\title{
COMUNICAÇÃO
}

\section{COROA DO ABACAXI 'SMOOTH CAYENNE' NA PRODUÇÃO DE MUDAS DO TIPO REBENTÃO}

\author{
'Smooth Cayenne' pineapple crown on the yield of suckers type planting material \\ Ruimário Inácio Coelho ${ }^{1}$, Almy Júnior Cordeiro de Carvalho², Jose Carlos Lopes ${ }^{1}$, \\ Silvio Lopes Teixeira ${ }^{3}$, Claudia Sales Marinho
}

\begin{abstract}
RESUMO
Com o objetivo de avaliar o emprego da coroa na produção de mudas do tipo rebentão, coroas do abacaxizeiro 'Smooth Cayenne', pesando de 200 a 300 gramas, foram cultivadas em vasos com $5 \mathrm{dm}^{3}$ de substrato, em casa de vegetação. Aos quinze e aos trinta dias após o plantio, foram pulverizadas com $10 \mathrm{~mL}$ de solução contendo $\mathrm{GA}_{3}\left(0,100,200\right.$ e $\left.400 \mathrm{mg} \mathrm{L}^{-1}\right)$ e BAP $(0,100,200$ e $400 \mathrm{mg} \mathrm{L}^{-1}$ ) em uma combinação das diferentes concentrações, totalizando 16 tratamentos e quatro repetições. O delineamento foi em blocos casualizados, no esquema fatorial 4x4. Observou-se efeito do GA 3 e do BAP na emissão de brotos, a cada período de dois meses, sendo que os maiores valores estimados foram de 1,81 brotos a cada 60 dias e um total médio de 12,6 brotos em 420 dias após o plantio, quando se utilizou $230 \mathrm{mg} \mathrm{L}^{-1}$ de BAP. Não houve efeito do $\mathrm{GA}_{3}$ e do BAP sobre o número, peso e comprimento dos maiores brotos colhidos aos 124, 214, 314 e 360 dias. Foram colhidas, em um período de 360 dias após o plantio da coroa, 5,2 mudas com peso médio de $145 \mathrm{~g}$ e comprimento igual a $35,9 \mathrm{~cm}$.
\end{abstract}

Termos para indexação: Ananas comosus, propagação, reguladores de crescimento, abacaxizeiro.

\begin{abstract}
Aiming to evaluate the use of 'Smooth Cayenne' pineapple plant crowns on yielding planting material of the suckers type, pineapple crowns, weighting from 200 to $300 \mathrm{~g}$, were planted in pots containing $5 \mathrm{dm}^{3}$ of substrate, in a greenhouse. Fifteen and thirty days after being planted, they were pulverized with $10 \mathrm{~mL}$ of GA $\left(0,100,200\right.$ and $\left.400 \mathrm{mg} \mathrm{L}^{-1}\right)$ and BAP $(0,100,200$ and $400 \mathrm{mg}$ $\left.\mathrm{L}^{-1}\right)$ combining the concentrations totalizing 16 treatments with four replicates. The experimental outline was in randomized blocks in a $4 \times 4$ factorial scheme. Effects of $\mathrm{GA}_{3}$ and $\mathrm{BAP}$ on shoot emission were evaluated every two-month intervals. The highest rates of shoot emission were 1,81 shoots every 60 days and a total average of 12.6 shoots at 420 days after planting, under $230 \mathrm{mg} \mathrm{L}^{-1}$ of BAP. There were no $\mathrm{GA}_{3}$ or BAP effects on the number, weight and length of the largest offshoots harvested at 124, 214,314 and 360 days. In a 365-day-period after planting, an average of 5.2 offshoots were harvested, with $145 \mathrm{~g}$ of average weight and $35.9 \mathrm{~cm}$ in length.
\end{abstract}

Index terms: Ananas comosus, propagation, growth regulators, pineapple plant.

\section{(Recebido em 10 de janeiro de 2007 e aprovado em 25 de abril de 2007)}

A coroa do abacaxi emerge do meristema apical após o florescimento e se desenvolve durante a formação do fruto, paralisando seu crescimento com a sua maturação, só retomando o crescimento quando plantada. Usada como muda, proporciona uma plantação homogênea, com florescimento e produção de frutos uniformes. Porém, como características desfavoráveis, apresenta um crescimento mais lento e frutificação mais tardia em relação aos outros tipos de mudas (DDUNGU, 1973; GIACOMELLI, 1982).

Gemas obtidas de coroa têm sido utilizadas como explantes, na micropropagação do abacaxizeiro (PASQUAL et al., 1998), e têm apresentado baixa taxa de variação somaclonal, quando comparadas aos explantes originados do sincarpo ou de gemas axilares (DAL-VESCO et al., 2000). A coroa também pode ser empregada na produção de mudas pelo método de seccionamento do caule (GIACOMELLI, 1982; REINHARDT \& CUNHA, 1999).

Uma coroa, dependendo do seu tamanho, pode apresentar até 40 folhas, podendo desenvolver dezenas de gemas, que, por sua vez, poderá gerar vários brotos. Portanto, há a possibilidade do uso da coroa para produção de mudas do tipo rebentão, não pelo método de

\footnotetext{
'Engenheiros Agrônomos, Doutores, Professores Associado - Departamento Produção Vegetal/DPV - Universidade Federal do Espírito Santo/UFES Alto Universitário s/n - Guararema - Cx. P. 16 - 29500-000 - Alegre, ES - ruimario@cca.ufes.br; jclopes@cca.ufes.br

'Engenheiros Agrônomos, Doutores, Professores - Laboratório de Fitotecnia/LFIT - Universidade Estadual do Norte Fluminense 'Darcy Ribeiro'/UENF _ Alberto Lamêgo, 2000 - Parque Califórnia - 28013-602 - Campos dos Goytacazes, RJ - almy@uenf.br; marinho@uenf.br

${ }^{3}$ Engenheiro Agrônomo, Ph.D, Professor - Laboratório de Fitotecnia/LFIT - Universidade Estadual do Norte Fluminense 'Darcy Ribeiro'/UENF - Alberto Lamêgo, 2000 - Parque Califórnia - 28013-602 - Campos dos Goytacazes, RJ - silvioteixeira@uenf.br
} 
seccionamento do caule, mas pelo seu plantio direto com destruição mecânica de seu ápice caulinar.

No Sri Lanka, onde a falta de mudas de qualidade é citada como a maior barreira à expansão da produção de abacaxi, a decapitação mecânica da gema apical é apontada como um método adequado para a multiplicação rápida do abacaxizeiro (HEENKENDA, 1993).

Souza et al. (2003) observaram um acentuado decréscimo no nível de AIA (309\%) em segmento nodal decapitado de Ananas comosus (L.) Merl., nas quatro primeiras horas de cultivo "in vitro", em meio livre de hormônios, período em que teve início a divisão celular na axila foliar. Comparativamente, a taxa auxinas/ citocininas total foi sempre menor no segmento nodal decapitado durante o processo de desenvolvimento das gemas axilares.

Objetivou-se com esta pesquisa foi avaliar o emprego da coroa do abacaxizeiro 'Smooth Cayenne' na produção de mudas tipo rebentão, utilizando o método de destruição mecânica da gema apical e o tratamento com ácido giberélico $\left(\mathrm{GA}_{3}\right)$ e benzilaminopurina (BAP).

$\mathrm{O}$ experimento foi desenvolvido em casa de vegetação instalada no Campus da Universidade Estadual do Norte Fluminense Darcy Ribeiro (UENF), utilizando coroas de abacaxizeiro colhidas em uma lavoura comercial. Depois de selecionadas por peso (200 a $300 \mathrm{~g}$ ), as coroas tiveram seus meristemas eliminados manualmente, com o auxílio de um alicate com ponta fina e uma chave de fenda.

Após a eliminação dos meristemas, as coroas foram tratadas, por imersão, em uma solução contendo benomil na concentração de $750 \mathrm{mg} \mathrm{L}^{-1}$ e parathion methyl $60 \mathrm{CE}$ na base de $0,95 \mathrm{~mL} \mathrm{~L}^{-1}$ de água, por cinco minutos, e plantadas no dia seguinte $(28 / 02 / 03)$ em vasos previamente preparados, onde foram colocados $5 \mathrm{dm}^{3}$ de substrato, preparado com terra de superfície + areia grossa lavada + esterco bovino + substrato comercial plantmax ${ }^{\hat{a}}$ para hortaliças nas proporções de 40:20: 20:20 (v/v), respectivamente. Os resultados das análises da amostra encontram-se na Tabela 1.

A adubação básica utilizada foi: $1,5 \mathrm{~g}$ de N, 1,0 $\mathrm{g}$ de $\mathrm{P}_{2} \mathrm{O}_{5}, 750 \mathrm{mg}$ de $\mathrm{K}_{2} \mathrm{O} ; 2,5 \mathrm{mg}$ de $\mathrm{B} ; 7,5 \mathrm{mg}$ de $\mathrm{Cu} ; 25 \mathrm{mg}$ de Fe e $25 \mathrm{mg}$ de $\mathrm{Zn}$ por vaso (MALAVOLTA, 1980), utilizandose como fontes a uréia, superfosfato simples, cloreto de potássio, para macronutrientes, e ácido bórico, sulfato de cobre, sulfato de zinco e sulfato ferroso para micronutrientes. Aos cento e vinte dias após o plantio, as coroas foram adubadas com $5 \mathrm{~g}$ de uréia.

Foi utilizado um esquema fatorial $4 \times 4$, no delineamento em blocos casualizados, em um total de 16 tratamentos. Quando foram consideradas as épocas de avaliação na análise estatística, estas compuseram as subparcelas, num esquema de parcelas subdivididas no tempo. Os tratamentos consistiram das concentrações, em mg L ${ }^{-1}$, de BAP $\left(0,100,200\right.$ e 400) e $\operatorname{GA}_{3}(0,100$, 200 e 400).

Foram preparadas soluções estoques para cada um dos reguladores de crescimento na concentração de 5000 $\mathrm{mg} \mathrm{L}^{-1}$, que foram utilizadas na preparação das soluções finais de acordo com os tratamentos. No preparo das soluções estoque, empregou-se o $\mathrm{HCl}$ a $0,1 \mathrm{~N}$ para a dissolução do BAP, na proporção de três gotas para cada $10 \mathrm{mg}$ do regulador, de acordo com Caldas et al. (1998). Como fonte do $\mathrm{GA}_{3}$ foi utilizado o produto comercial ProGibbÒ, contendo $10 \%$ da substância ativa. $\mathrm{O}$ pH das soluções foi ajustado para 5,6.

Aos quinze dias após o plantio (15/03/2003), as coroas receberam o primeiro tratamento com $\mathrm{GA}_{3}$ e BAP, via foliar, por meio de pulverização com $10 \mathrm{~mL}$ de solução, nas respectivas concentrações de acordo com os tratamentos. A aplicação foi repetida quinze dias após a primeira. Para aplicação dos reguladores, utilizou-se um borrifador manual com capacidade de $500 \mathrm{~mL}$.

Durante a condução do experimento, as plantas receberam, preventivamente, apenas uma pulverização com fungicida, não tendo sido registrada ocorrência de doença.

As avaliações foram feitas pela contagem de brotos emitidos a intervalos bimestrais durante 420 dias, considerando-se número, peso e comprimento médio dos brotos igual ou maior que $20 \mathrm{~cm}$ colhidos aos 124, 214, 314 e 360 dias após o plantio das coroas. Avaliou-se, ainda, o efeito dos reguladores de crescimento sobre as seguintes características das coroas: número de folhas, diâmetro e comprimento dos caules das coroas, ao final do experimento.

As primeiras brotações foram registradas aos 33 dias após o plantio das coroas, por causa do efeito da eliminação do ápice caulinar na liberação das gemas axilares da dominância apical (LI et al., 1995). Heenkenda (1997), avaliando a emissão de rebentões pelo abacaxizeiro 'Kew', empregando o método de decapitação mecânica, observou a emissão de brotos a partir de 58 dias após o tratamento. Porém, este utilizou plantas em estádio de desenvolvimento bem mais avançado.

A análise de regressão para número de brotos emitidos revelou efeito significativo do $\mathrm{GA}_{3}$ e do BAP, em um comportamento quadrático (Figura 1). A maior média estimada para número total de brotos emitidos durante o período do experimento foi de 12,6 brotos para coroas tratadas com 230,5 $\mathrm{mg} \mathrm{L}^{-1}$ de BAP ou 201,5 $\mathrm{mg} \mathrm{L}^{-1} \mathrm{de}_{\mathrm{GA}_{3}}$ (Figura 1). 
Tabela 1 - Resultados das análises químicas da amostra do substrato.

\begin{tabular}{|c|c|c|c|c|c|c|c|c|c|c|c|c|c|c|}
\hline $\mathrm{pH}$ & $\mathrm{K}$ & $\mathrm{Ca}$ & $\mathrm{Mg}$ & $\mathrm{Al}$ & $\mathrm{H}+\mathrm{Al}$ & S.B & $\mathrm{V}$ & $\mathrm{P}$ & $\mathrm{Fe}$ & $\mathrm{Cu}$ & $\mathrm{Zn}$ & $\mathrm{Mn}$ & $S$ & $\mathrm{~B}$ \\
\hline 0 & \multicolumn{6}{|c|}{$\mathrm{cmol}_{\mathrm{c}} \mathrm{dm}^{-3}$} & $(\%)$ & \multicolumn{7}{|c|}{$\mathrm{mg} \mathrm{dm}^{-3}$} \\
\hline & 4,4 & 3,4 & 2,0 & 0,0 & 4,4 & 5,9 & 57 & 16 & 58 & 0,2 & 5,9 & 24,5 & 55,4 & 0,41 \\
\hline
\end{tabular}

Matéria orgânica $=38,1 \mathrm{~g} \mathrm{dm}^{-3}$

Análises realizadas na UFRRJ/Campus Leonel Miranda, Campos dos Goytacazes, RJ.

Naqvi (1999), Normanly (1997) e Raven et al. (1999), afirmam que os reguladores de crescimento raramente agem isoladamente, atuando em conjunto com outros reguladores internos do crescimento e do desenvolvimento. Porém, nesta pesquisa, não foi observado efeito significativo para interação entre $\mathrm{GA}_{3}$ e BAP, em aplicação exógena, sobre a brotação das gemas axilares das coroas do abacaxizeiro 'Smooth Cayenne'. Também não foi observado efeito das interações entre reguladores de crescimento e época de avaliação, comportamento que pode ter explicação na freqüência e no período de aplicação destes reguladores.

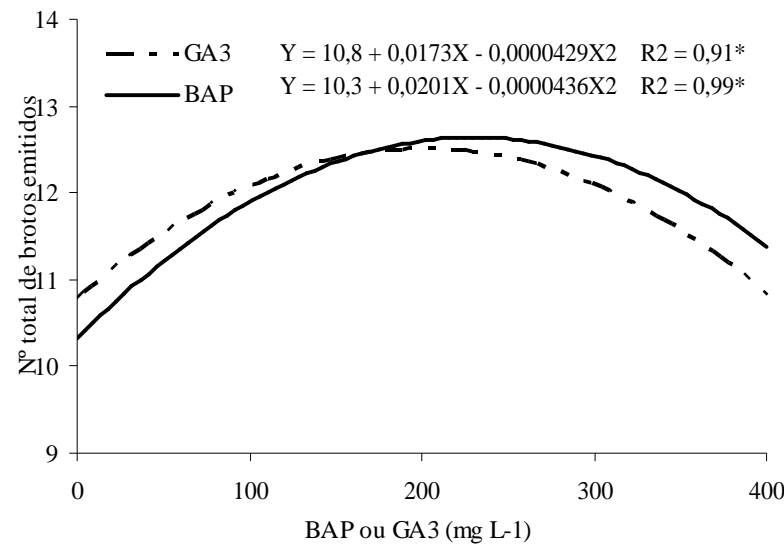

Figura 1 - Número total de brotos emitidos por coroa do abacaxizeiro 'Smooth Cayenne' em 420 dias, em função de BAP e de GA.

Observou-se efeito do período de avaliação, em intervalos de 60 dias até 420 dias após o plantio das coroas, sobre o número de brotos emitidos (Tabela 2). Acredita-se que as colheitas dos brotos iguais ou maiores do que 20 $\mathrm{cm}$ aos 124, 234 e 314 dias após o plantio, possam estar associadas ao comportamento observado para número de brotos emitidos pelas coroas, em função da época de avaliação.

Com a retirada dos brotos maiores, eliminou-se, temporariamente, o principal sítio de síntese de auxinas (MAERERE, 1997; SOUZA et al., 2003), diminuindo, assim, a relação endógena de auxinas/citocininas nos tecidos, estimulando a brotação das gemas axilares. Isto pode explicar o comportamento observado, uma vez que não houve uma sincronia ou regularidade entre as épocas de colheita e as épocas de avaliação dos brotos emitidos.

Adaniya et al. (2004) observaram que a remoção dos brotos com $5 \mathrm{~cm}$, em secções de caule do abacaxizeiro 'Smooth Cayenne' com $12 \mathrm{~cm}$ de comprimento sem corte longitudinal, tratadas com $5 \mathrm{mg} \mathrm{L}^{-1}$ de CPPU, além de aumentar o número de brotos emitidos, aumentou a uniformidade dos brotos em altura.

As análises dos dados para número de brotos colhidos, comprimento e peso médio dos brotos por coroa aos 124, 234, 314 e aos 360 dias após o plantio das secções, não demonstraram efeitos significativos em função dos tratamentos com $\mathrm{GA}_{3}$ e BAP. Porém, observou-se diferenças significativas em função da época de colheita para estas variáveis (Tabela 3 ).

As diferenças estatísticas observadas para comprimento e peso médio do maior broto em função da época são explicadas pelo maior ou menor intervalo entre as datas das colheitas. Observa-se, na Tabela 3, que os maiores valores para comprimento e pesos médios dos brotos foram registrados nas avaliações após 234 e 314 dias, portanto, com os maiores intervalos entre colheitas, não sendo observadas diferenças significativas para estas características entre os brotos colhidos nas duas épocas.

O menor peso médio registrado para os brotos colhidos aos 360 dias, além do curto espaço de tempo em relação a última colheita, provavelmente, sofreu alguma influência pelo maior número de brotos colhidos, como pode ser visualizado na Tabela 2 , onde também se observa que o peso médio dos maiores brotos é o dobro da média geral.

O número médio para o total de brotos colhidos com comprimento médio de $35,86 \mathrm{~cm}$, de 5,3 brotos por coroa, no período de 360 dias, pode ser considerado bom, levando-se em conta que aos 124 dias após o plantio foi colhido em média 1,0 broto por coroa. Nenhum resultado semelhante foi relatado por outro método de multiplicação 
Tabela 2 - Número médio de brotos emitidos em função da época de avaliação após o plantio das coroas do abacaxizeiro 'Smooth Cayenne'*.

\begin{tabular}{cc}
\hline Períodos de avaliação (dias após o plantio) & Número de brotos emitidos \\
\hline $0-60$ & $0,867 \quad \mathrm{c}$ \\
$60-120$ & $1,945 \mathrm{~b}$ \\
$120-180$ & $1,421 \mathrm{bc}$ \\
$180-240$ & $1,867 \mathrm{~b}$ \\
$240-300$ & $0,898 \mathrm{c}$ \\
$300-360$ & $1,117 \mathrm{c}$ \\
$360-420$ & $3,430 \mathrm{a}$ \\
\hline Média & 1,649 \\
\hline CV $(\%)$ & 70,9 \\
\hline
\end{tabular}

*Médias seguidas da mesma letra nas colunas não difere entre si ao nível de 5\% de probabilidade pelo teste de Tukey.

Tabela 3 - Número médio de brotos colhidos, peso e comprimento médio de todos os brotos colhidos (média geral) e média do maior broto por coroa do abacaxizeiro 'Smooth Cayenne'*.

\begin{tabular}{|c|c|c|c|c|c|}
\hline \multirow{2}{*}{$\begin{array}{c}\text { Períodos de } \\
\text { avaliação (dias } \\
\text { após o plantio) }\end{array}$} & \multirow{2}{*}{$\begin{array}{c}\text { Número de } \\
\text { brotos } \\
\text { colhidos }\end{array}$} & \multicolumn{2}{|c|}{ Peso de broto $(\mathrm{g})$} & \multicolumn{2}{|c|}{ Comprimento de broto $(\mathrm{cm})$} \\
\hline & & $\begin{array}{l}\text { Média geral } \\
\text { (TBC) }\end{array}$ & $\begin{array}{l}\text { Média do maior } \\
\text { broto }\end{array}$ & $\begin{array}{l}\text { Média geral } \\
\text { (TBC) }\end{array}$ & $\begin{array}{l}\text { Média do maior } \\
\text { broto }\end{array}$ \\
\hline 124 & $1,08 \mathrm{~b}$ & $118 b$ & $137 \mathrm{~b}$ & $29,7 \mathrm{c}$ & $31,7 \mathrm{~b}$ \\
\hline 234 & $0,96 \mathrm{~b}$ & $184 \mathrm{a}$ & $221 \mathrm{a}$ & $36,6 \mathrm{~b}$ & $42,0 \mathrm{a}$ \\
\hline 314 & $0,85 \mathrm{~b}$ & $194 \mathrm{a}$ & $222 \mathrm{a}$ & $42,2 \mathrm{a}$ & $44,2 \mathrm{a}$ \\
\hline 360 & $2,31 \mathrm{a}$ & $83 \mathrm{c}$ & $163 \mathrm{~b}$ & $34,9 \mathrm{~b}$ & $41,9 \mathrm{a}$ \\
\hline Média & 1,30 & 145 & 186 & 35,9 & 40,0 \\
\hline $\mathrm{CV}(\%)$ & 53,7 & 40,1 & 40,2 & 17,0 & 17,7 \\
\hline
\end{tabular}

* Médias seguidas da mesma letra nas colunas não difere entre si ao nível de 5\% de probabilidade pelo teste de Tukey.

do abacaxizeiro, observado o período decorrido entre o plantio da matriz e a colheita dos primeiros brotos. Braga \& Sá (2001), obtiveram maior rendimento, 3,2 brotos com peso superior a $100 \mathrm{~g}$, empregando caule de $20 \mathrm{~cm}$ fracionado em secções de $5 \mathrm{~cm}$, aos 10 meses após o plantio.

Os resultados observados nesta pesquisa demonstram o potencial das coroas na produção precoce de rebentões pelo método de destruição do meristema apical. Indicam, ainda, a possibilidade de que o número de brotos emitido e colhido possa aumentar com a retirada constante do maior broto, a intervalos mais curtos, porém, o tamanho mínimo para colheita dos brotos ainda precisa ser definido. $\mathrm{O}$ que deve ser feito em função do destino que se daria aos brotos, se para o plantio direto no campo ou seu transplante para viveiro.
Os resultados observados levam-nos a concluir que:

- $\mathrm{O} \mathrm{GA}_{3}$ e o BAP influenciam o número de brotos emitidos pelas coroas;

- a aplicação de BAP na concentração de $230 \mathrm{mg} \mathrm{L}$ ${ }^{1}$ determina maior emissão de brotos por coroa 12,67 brotos;

- em um período de 360 dias após o plantio da coroa, pode-se colher 5,2 mudas com média de peso igual a $145 \mathrm{~g}$ e de comprimento igual a $35,9 \mathrm{~cm}$;

- os tratamentos com $\mathrm{GA}_{3}$ e BAP não apresentam efeitos sobre o número, comprimento e peso de brotos colhidos até 360 dias após o plantio da coroa, verificandose, entretanto, efeito da época de colheita dos brotos sobre estas características;

- a coroa pode se constituir em uma alternativa para a produção precoce de muda tipo rebentão, pela eliminação do ápice caulinar. 


\section{REFERÊNCIAS BIBLIOGRÁFICAS}

ADANIYA, S.; MINEMOTA, K.; MOROMIZATO, Z.; MOLOMURA, K. The use of CPPU for efficient propagation of pineapple. Scientia Horticultura, Amsterdam, v. 100, p. 7-14, 2004.

BRAGA, M. F.; SÁ, M. E. L. Smooth Cayenne pineapple propation by stem sections. Revista Brasileira de Fruticultura, Jaboticabal, v. 23, n. 1, p. 175-178, 2001.

CALDAS, L. S.; HARIDOSAN, P.; FERREIRA, M. E. Meios nutritivos. In: TORRES, A. C.; CALDAS, L. S.; BUSO, J. A. (Eds.). Cultura de tecidos e transformação genética de plantas. Brasília, DF: Embrapa-SPI, 1998. p. 371-394.

DAL-VESCO, L. L.; PESCADOR, R.; BELÔ, A.; FEUSA, S.; OLIVEIRA, E. N.; BRANCHER, A.; ZAFFARI, B. R.; NODARI, R. O.; GUERRA, P. Qualidade genotípica de mudas e performance a campo de plantas micropropagadas de abacaxizeiro. Revista Brasileira de Fruticultura, Jaboticabal, v. 22, n. 1, p. 80-85, 2000.

DDUNGU, J. C. M. Rooting-behaviour of diferent types of peneapple propagating material. Acta Horticulturae, The Hague, v. 33, p. 155-159, 1973.

GIACOMELLI, E. J. Expansão da abaxicultura no Brasil. Campinas: Fundação Cargill, 1982. 79 p.

HEENKENDA, H. M. S. Effect of plant size on sucker promotion in 'Mauritius' pineapple by mechanical decapitation. Acta Horticulture, The Hague, v. 334, p. 331336, 1993.

HEENKENDA, H. M. S. Effect of plant density and establishment method on sucker promotion in 'Kew' pineapple by mechanical decapitation. Acta Horticulture, The Hague, v. 425, p. 321-328, 1997.
LI, C. J.; GUEVARA, E.; HERRERA, J.; BANGERTH, F. Effect of apex excision and replacement by 1naphthylacetic acid on cytokinin concentracion and apical dominance in pea plants. Physiology Plantarum, Copenhagen, v. 94, p. 465-469, 1995.

MAERERE, A. P. Axillary-bud development as it determines suckering in 'Queen Victoria' and 'Smooth Cayenne' pineapples. Acta Horticulture, The Hague, v. 425, p. 309320, 1997.

MALAVOLTA, E. Elementos de nutrição mineral de plantas. Piracicaba: Ceres, 1980. $251 \mathrm{p}$.

NAQVI, S. S. M. Plant hormones and stress phenomena. In: PESSARAKLI, M. (Ed.). Handbook of plant and crop stress. New York: M. Dekker, 1999. p. 709-730.

NORMALY, J. Auxin metabolismo.Physiologia Plantarum, Copenhagen, v. 100, p. 431-442, 1997.

PASQUAL, M.; MOREIRA, M. A.; ALVES SOBRINHO, A. Biotecnologia aplicada à produção de mudas de abacaxi. Informe Agropecuário, Belo Horizonte, v. 19, n. 195, p. 20 23, 1998.

RAVEN, P. H.; EVERT, R. E.; EICHHORN, S. E. Fisiologia vegetal. 6. ed. Washington, DC: W.H. Freeman, 1999. 906 p.

REINHARDT, H. R. C.; CUNHA, G. A. P. Métodos de programação. In: CABRAL, J. R. S.; SOUZA, L. F. da S. O abacaxizeiro: cultivo, agroindústria e economia. Brasília, DF: Embrapa Mandioca e Fruticultura, 1999. p. 105-138.

SOUZA, B. M.; KRAUS, J. E.; ENDRES, L.; MERCIER, H. Relationships between endogenous hormonal levels and axillary bud development of Ananas comosus nodal segments. Plant Physiology and Biochemistry, [S.1.], v. 41, p. 733-739, 2003. 